Claremont Colleges

Scholarship@ Claremont

All HMC Faculty Publications and Research

HMC Faculty Scholarship

$1-1-1979$

\title{
A Semilinear Dirichlet Problem
}

Alfonso Castro

Harvey Mudd College

\section{Recommended Citation}

Castro, Alfonso. “A semilinear Dirichlet problem” Canadian J. of Math., Vol. XXXI, No. 2(1979), pp. 337-340.

This Article - preprint is brought to you for free and open access by the HMC Faculty Scholarship at Scholarship @ Claremont. It has been accepted for inclusion in All HMC Faculty Publications and Research by an authorized administrator of Scholarship @ Claremont. For more information, please contact scholarship@cuc.claremont.edu. 


\title{
A SEMILINEAR DIRICHLET PROBLEM
}

\author{
ALFONSO CASTRO
}

Introduction and notations. Let $\Omega$ be a bounded region in $\mathbf{R}^{n}$. In this note we discuss the existence of weak solutions (see [4, Section 2]) of the Dirichlet problem

$$
\text { (I) } \begin{array}{rl}
\Delta u(x)+g(x, u(x))+f(x, u(x), \nabla u(x))=0 & x \in \Omega \\
u(x) & =0 \quad x \in \partial \Omega
\end{array}
$$

where $\triangle$ is the Laplacian operator, $g: \Omega \times \mathbf{R} \rightarrow \mathbf{R}$ and $f: \Omega \times \mathbf{R}^{n+1} \rightarrow \mathbf{R}$ are functions satisfying the Caratheodory condition (see [2, Section 3]), and $\nabla$ is the gradient operator.

We let $\lambda_{1}<\lambda_{2} \leqq \ldots \leqq \lambda_{m} \leqq \ldots$ denote the sequence of numbers for which the problem

$$
\begin{aligned}
\triangle u(x)+\lambda u(x) & =0 & & x \in \Omega \\
u(x) & =0 & & x \in \Omega
\end{aligned}
$$

has nontrivial weak solutions.

The main result of this paper is:

Suppose the following two hypotheses hold. (1.1) The function $g(x, u)$ admits a derivative with respect to $u, \partial g / \partial u: \Omega \times \mathbf{R} \rightarrow \mathbf{R}$, which satisfies the Caratheodory condition; furthermore, there exist $\alpha, \alpha_{1} \in \mathbf{R}$ and a positive integer $N$ such that

$$
\lambda_{N}<\alpha \leqq \partial g / \partial u(x, u) \leqq \alpha_{1}<\lambda_{N+1} \text { for all }(x, u) \in \Omega \times \mathbf{R}
$$

(1.2) There exist a constant $\beta>0$ and a function $c(x) \in L_{2}(\Omega)$ such that

$$
|f(x, u, y)|^{2} \leqq c(x)+\beta^{2}\|y\|^{2}
$$

for all $(x, u, y) \in \Omega \times \mathbf{R} \times \mathbf{R}^{n}$, where \|\| denotes the usual norm in $\mathbf{R}^{n}$. If

$$
\beta<\left(\min \left\{1-\alpha_{1} / \lambda_{N+1}, \alpha / \lambda_{N}-1\right\}\right) / \sqrt{\lambda_{1}}
$$

then (I) has a weak solution.

As a corollary of our main result we obtain bounds for the eigenvalues on $\left(\lambda_{N}, \lambda_{N+1}\right)$ of a class of non-selfadjoint problems of the form:

$$
\text { (III) } \begin{array}{rl}
\triangle u(x)+\left\langle\left(a_{1}(x), \ldots, a_{n}(x)\right), \nabla u(x)\right\rangle+\lambda u(x)=0 & x \in \Omega \\
u(x) & =0 \quad x \in \partial \Omega,
\end{array}
$$

where $\langle$,$\rangle denotes the usual inner product in \mathbf{R}^{n}$ and $a_{1}, \ldots, a_{n} \in L_{\circ}(\Omega)$.

Received November 3, 1977 and in revised form March 22, 1978. This research was done while the author was a Lefschetz researcher at Centro de Investigacion del IPN. 
In $[\mathbf{2}$, Theorem 1] and $[\mathbf{4}$, Theorem 3.1] the problem (I) is considered and the existence of weak solutions is proved when $f(x, u, y)=o(\|y\|)$ as $\|y\| \rightarrow+\infty$. In [3, Theorem 3.4] the problem (I) is studied when $\Omega \subset \mathbf{R}$ and $f$ and $g$ are permitted to depend on the second order derivatives. The results of [3] yield inequalities of the form (1.3) when $\alpha_{1}<\lambda_{1}$. We denote in this paper by $H^{1}$ the Sobolev space $H_{0}{ }^{1,2}(\Omega)$ (see [1, p. 45]). We take as inner product in $H^{1}$ the bilinear form defined by

$$
\langle u, v\rangle_{1}=\int_{\Omega}\langle\nabla u(\xi), \nabla v(\xi)\rangle d \xi .
$$

We denote by \|\| the norm on $H^{1}$ and by \|\|$_{0}$ the norm on $L_{2}(\Omega)$. We let $X$ denote the closed subspace of $H^{1}$ spanned by the eigenfunctions of (II) corresponding to eigenvalues $\lambda_{k}$ with $\lambda_{k} \leqq \lambda_{N}$. We use the symbol $\int$ to mean integral over $\Omega$.

Proofs. From now on we assume that (1.1) and (1.2) hold. Let $J: H^{1} \times H^{1} \rightarrow \mathbf{R}$ be defined by

$$
J(y, u)=\int\left\{\|\nabla u(\xi)\|^{2} / 2-G(\xi, u(\xi))-f(\xi, y(\xi), \nabla y(\xi)) u(\xi)\right\} d \xi,
$$

where $G: \Omega \times \mathbf{R} \rightarrow \mathbf{R}$ is a continuous function such that $\partial G / \partial u(x, u)=g(x, u)$ and $G(x, 0)=0$. It is not difficult to see that for $y, u, v \in H^{1}$

$$
\begin{array}{r}
\lim _{t \rightarrow \infty}(J(y, u+t v)-J(y, u)) / t=\int\{\langle\nabla u(\xi), \nabla v(\xi)\rangle-g(\xi, u(\xi)) v(\xi) \\
-f(\xi, y(\xi), \nabla y(\xi)) v(\xi)\} d \xi .
\end{array}
$$

Therefore, by Vainberg's lemma (see [6, p. 63]), if (1.1) holds, the right hand side of (2.1) defines a continuous linear functional on $v \in H^{1}$. Hence, for each $(y, u) \in H^{1} \times H^{1}$ there exist $S(y, u) \in H^{1}$ such that

$$
\lim _{t \rightarrow 0} J(y, u+t v)-J(y, u) / t=\langle u, v\rangle_{1}+\langle S(y, u), v\rangle_{1} .
$$

By (1.1), $(1,2)$ and Vainberg's Lemma (see [2, Proposition 4]) the functions $u(\xi) \rightarrow g(\xi, u(\xi))$ and $y(\xi) \rightarrow f(\xi, y(\xi), \nabla y(\xi))$ are continuous functions from $H^{1}$ into $L_{2}(\Omega)$. Since, by Rellich's principle, the inclusion of $L_{2}(\Omega)$ into the dual space of $H^{1}$ is compact, $S(y, u)$ is a compact function.

From (1.1) and the results of [5, Section 7] it follows that

$$
\begin{aligned}
\triangle u(x)+g(x, u(x))+f(x, y(x), \nabla y(x)) & =0 & & x \in \Omega \\
u(x) & =0 & & x \in \partial \Omega
\end{aligned}
$$

has a unique weak solution for each $y \in H^{1}$. Therefore, for each $y \in H^{1}$ there exists a unique $\varphi(y) \in H^{1}$ such that

$$
\langle\varphi(y)+S(y, \varphi(y)), v\rangle_{1}=0 \text { for all } v \in H^{1} .
$$


Lemma 1. The function $\varphi: H^{1} \rightarrow H^{1}$ defined by (2.3) is compact.

Proof. First we show that $\varphi$ is continuous. From the discussion in $[\mathbf{5}$, Section 7] we see that if $J_{y}: H^{1} \rightarrow \mathbf{R}$ is defined by $J_{y}(u)=J(y, u)$ then $J_{y}$ is of class $C^{2}$. Let $D J_{y}(u)$ be the Hessian of $J_{y}$ at $u$. An elementary computation show that

$$
\left\langle D J_{y}(u) v, v\right\rangle_{1}=\int\left\{\|\nabla v\|^{2}-\partial g / \partial u(\xi, u(\xi)) v^{2}(\xi)\right\} d \xi
$$

Following the arguments of $[\mathbf{4}$, Section 7$]$ we see that $D J_{y}(u)$ is a nonsingular Fredholm operator.

Let $T: H^{1} \times H^{1} \rightarrow H^{1}$ be defined by $T(y, u)=u+S(y, u)$. Hence $T$ is continuously differentiable with respect to $u$ and $\partial_{u} T(y, u)=D J_{Y}(u)$. Thus, by $(2.3)$, for any $y_{0} \in H^{1} T\left(y_{0}, \varphi\left(y_{0}\right)\right)=0$. By the foregoing argument $\partial_{u} T\left(y_{0}, \varphi\left(y_{0}\right)\right)$ is nonsingular. Therefore, by the implicit function theorem there exist a neighborhood $V$ of $y_{0}$ and a continuous function $\psi: V \rightarrow H^{1}$ such that $T(y, \psi(y))=0$ for all $y \in V$. Consequently, by the uniqueness of $\varphi(y)$, we have $\varphi(y)=\psi(y)$ on $V$, and this proves that $\varphi$ is continuous.

Now we prove that $\varphi$ is bounded on bounded sets. For $y \in H^{1}$, let $\varphi_{1}(y)$ be the orthogonal projection of $\varphi(y)$ on $X$, and let $\varphi_{2}(y)$ be $\varphi(y)-\varphi_{1}(y)$. By (2.3) we have

$$
0=\left\langle\varphi(y)+S(y, \varphi(y)), \varphi_{2}(y)-\varphi_{1}(y)\right\rangle_{1} .
$$

Hence,

$$
\begin{array}{r}
0=\left\|\varphi_{2}(y)\right\|_{1}^{2}-\left\|\varphi_{1}(y)\right\|_{1}^{2}-\int g(\xi, \varphi(y)(\xi))\left(\varphi_{2}(y)(\xi)-\varphi_{1}(y)(\xi)\right) d \xi \\
\quad-\int f(\xi, y(\xi), \nabla y(\xi))\left(\varphi_{2}(y)(\xi)-\varphi_{1}(y)(\xi)\right) d \xi \\
\geqq\left\|\varphi_{2}(y)\right\|_{1}^{2}-\left\|\varphi_{1}(y)\right\|_{1}^{2}-\sqrt{\lambda_{1}}|| g(\xi, 0)\left\|_{0} \cdot\right\| \varphi(y)\left\|_{1}-\alpha_{1}\right\| \varphi_{2}(y) \|_{0}^{2} \\
\quad+\alpha\left\|\varphi_{1}(y)\right\|_{0}^{2}-\left(\int f^{2}(\xi, y(\xi), \nabla y(\xi)) d \xi\right)^{1 / 2} \cdot \sqrt{\lambda_{1}} \cdot \|\left.\varphi(y)\right|_{1} \\
\geqq\left(1-\alpha_{1} / \lambda_{N+1}\right)\left\|\varphi_{2}(y)\right\|_{1}^{2}+\left(\alpha / \lambda_{N}-1\right)\left\|\varphi_{1}(y)\right\|_{1}^{2} \\
-\sqrt{\lambda_{1}}\|g(\xi, 0)\|_{0}\|\varphi(y)\|_{1}-\left(\int f^{2}(\xi, y(\xi), \nabla y(\xi)) d \xi\right)^{1 / 2} \cdot \sqrt{\lambda_{1}} \cdot\|\varphi(y)\|_{1} .
\end{array}
$$

Thus, if $m=\min \left\{1-\alpha_{1} / \lambda_{N+1}, \alpha / \lambda_{N}-1\right\}$ then we have

$$
\sqrt{\lambda_{1}}\|g(\xi, 0)\|_{0}+\sqrt{\lambda_{1}}\left(\int f^{2}(\xi, y(\xi), \nabla y(\xi)) d \xi\right)^{1 / 2} \geqq m\|\varphi(y)\|_{1} .
$$

Since, by (1.2), the Nemytski operator $y(\xi) \rightarrow f(\xi, y(\xi), \nabla y(\xi))$ maps bounded sets of $H^{1}$ into bounded sets of $L_{2}(\Omega)$ we infer from (2.5) that $\varphi$ is bounded on bounded sets. 
Suppose $\left\{y_{n}\right\}$ is a bounded sequence in $H^{1}$. Hence $\left\{S\left(y_{n}, \varphi\left(y_{n}\right)\right\}\right.$ contains a convergent subsequence $\left\{S\left(y_{n_{j}}, \varphi\left(y_{n_{j}}\right)\right)\right\}$. By $(2.3),-\varphi\left(y_{n_{j}}\right)=S\left(y_{n_{j}}, \varphi\left(y_{n_{j}}\right)\right)$. Therefore, $\left\{\varphi\left(y_{n_{j}}\right)\right\}$ is a convergent sequence. Consequently, $\varphi$ is compact and the lemma is proved.

Theorem 2. If (1.1), (1.2) and (1.3) hold then the problem (I) has a weak solution.

Proof. By (1.2), there exists $K \in \mathbf{R}$ such that

$$
\left(\int f^{2}(\xi, y(\xi), \nabla y(\xi)) d \xi\right) \leqq K^{2}\|c(x)\|_{0}+\beta^{2}\|y\|_{1}^{2}
$$

for all $y \in H^{1}$. Combining (2.5) and (2.6) we have

$$
m\|\varphi(x)\|_{1} \leqq \sqrt{\lambda_{1}}\|g(\xi, 0)\|_{0}+\sqrt{\lambda_{1}} K\|c(x)\|_{0^{1 / 2}}+\beta \sqrt{\lambda_{1}}\|y\|_{1} .
$$

Therefore, by (1.3), if $R>0$ is big enough then the function $\varphi$ maps the ball of center 0 and radius $R$ into itself. Consequently, by Schauder's fixed point theorem, $\varphi$ must have a fixed point. Since any fixed point of $\varphi$ is a weak solution of (I) the theorem is proved.

Corollary 3. If $\left(\int\left(a_{1}{ }^{2}(\xi)+\ldots+a_{n}{ }^{2}(\xi)\right) d \xi\right)^{1 / 2} \leqq \beta$ then the problem (III) does not have eigenvalues in the open interval

$$
\left(\lambda_{N}\left(1+\beta \sqrt{\lambda_{1}}\right), \lambda_{N+1}\left(1-\beta \sqrt{\lambda_{1}}\right)\right)=D .
$$

Proof. If $\lambda \in D$, then following the proof of Theorem 2 we see that for any $c(x) \in L_{2}(\Omega)$ the problem

$$
\begin{aligned}
\Delta u(x)+\left\langle\left(a_{1}(x), \ldots, a_{n}(x)\right), \nabla u(x)\right\rangle+\lambda u(x) & =c(x) \quad x \in \Omega \\
u(x) & =0 \quad x \in \partial \Omega
\end{aligned}
$$

has a weak solution. Therefore by the Fredholm alternative (see [2, Proposition 1]) $\lambda$ cannot be an eigenvalue of (III).

\section{REFERENCES}

1. R. Adams, Sobolev spaces, (Academic Press, 1975).

2. D. J. de Figueiredo, The Dirichet problem for non-linear elliptic equations: a Hilbert space approach, Lecture Notes in Math. 446, (Springer, 1974).

3. P. M. Fitzpatrick, Existence results for equations involving noncompact perturbations of Fredholm mappings with applications to differential equations (mimeographed copy).

4. E. Landesman and A. C. Lazer, Linear eigenvalue problems and a nonlinear boundary value problem, Pacific J. of Math., 33 (1970), 311-328.

5. A. Lazer, E. Landesman and D. Meyers, On saddle point problems in the calculus of variations, the Ritz algorithm, and monotone convergence, J. Math. Anal. Appl., 53 (1975), 594-614.

6. M. Vainberg, Variational methods in the study of nonlinear operators, (Holden-Day, San Francisco, 1964).

Centro de Investigacion del IPN, México 